\title{
Multivariable Control of Multi-Zone Chemical Mechanical Polishing
}

\author{
Sheng-Jyh Shiul, Cheng-Ching Yu ${ }^{* 1}$, Shih-Haur Shen ${ }^{2}$, and An-Jhih Su ${ }^{1}$ \\ ${ }^{1}$ Dept. of Chem. Eng., National Taiwan UniversitTaipei 106-17, TAIWAN \\ ${ }^{2}$ Applied Materials Taiwan Ltd., Hsin-Chu 300, TAIWAN
}

\begin{abstract}
The modeling and multivariable control of the multi-zone CMP are studied in this work. In the process control notation, the manipulated variables are the three pressures applied to each zone. Therefore, this is a $60 \times 3$ non-square multivariable control problem. The singular value decomposition (SVD) is used to design a non-square feedback controller. The proposed control system is test on incoming wafers with different surface profiles. Results show that achievable performance can be maintained using the proposed SVD controller.
\end{abstract}

\section{INTRODUCTION}

Despite recent advances in CMP, some manufacturing concerns associated with successful implementation of CMP remain to be overcome. In theory, CMP can achieve global planarity, but there is still a problem that different operating conditions will result in non-uniformity in thickness of wafer surface. The within-wafer nonuniformity (WIWNU) indicates the variation in surface thickness across the wafer radial position, especially on the edge. Besides, the surface profile of wafers produced from electrochemical plating (ECP) process appears that the metallic layer is thicker on edge area. Thus, a new type of CMP, multi-zone CMP, offers an attractive alternative. Multi-zone CMP is expected to reduce WIWNU and to provide a wider processing window. Unlike the typical single zone configuration, the wafer carrier is divided into multiple zones in the radial position and different pressure can be applied to each zone (Fig. 1). The objective of this work is to devise a systematic approach to the modeling and control of such CMP processes.

\section{MULTI-ZONE CMP}

\subsection{Process Description.}

Consider a CMP system where the wafer carrier is divided into three zones in the radial position and different pressure can be applied to each zone (Fig. 1). For a wafer carrier with the radius of $150 \mathrm{~mm}$ (i.e., a $300 \mathrm{~mm}$ wafer), zone \#1 covers $0-130 \mathrm{~mm}$, the second zone ranges from 130 $140 \mathrm{~mm}$, and third zone covers $140 \mathrm{~mm}$ and beyond. Typically, the well-known Preston's equation [1] is used to model the polishing process. It describes the material removal as a linear function of pressure and rotation speed.

$$
R R=K_{p} \cdot p \cdot v
$$

corresponding author; e-mail: ccyu@ntu.edu.tw; fax: +886-22362-3040 where $K_{p}$ is the Preston constant, $p$ is pressure, and $v$ is rotation speed. The Preston equation can be extended to multi-zone CMP in a straightforward manner. For the multi-zone system, the relationship between removal rate at the ith radial position and input variables can be expressed as:

$$
R R_{i}=\sum_{j} K_{p, i, j} p_{j} \cdot v
$$

where $K_{p . i . j}$ is the local Preston constant describing the effect of pressure from $\mathrm{jth}$ zone on the ith radial position, $p_{j}$ denotes the pressure of the jth zone, and $v$ is again the rotation speed. Without loss of generality, assume that the rotation speed is fixed throughout all runs and $v$ is absorbed into $K_{p, i, j}$ for the subsequent development.

Copper (Cu) CMP is carried out on an Applied Materials' Reflexion $^{\mathrm{TM}}$ polisher using Titan Profiler $^{\mathrm{TM}}$ polishing heads. Polishing pad (Rodel IC1010) is used and the experimental copper CMP slurry is provided by Cabot Corporation. Electrochemical plating (ECP) process is employed for $\mathrm{Cu}$ plating. The $300 \mathrm{~mm}$ wafers without pattern are used in all tests. All copper thickness measurements are performed with an in situ i-Scan sensor.

All the experiments were carried out according to a standard design of experiment procedure (DOE). The factors in this work are the three pressures applied to each zone and a pressure applied to the retaining ring. From center of the wafer to the edge, they are defined as $p_{l}, p_{2}$, $p_{3}$, and $p_{r r}$, respectively. We devise two levels for each factor such that a four-factor and two-level design of experiment (DOE) is carried out.

\subsection{Steady-State Analysis}

From the definitions of state and input variables, the steady-state behavior for the multivariable system can be written as follows:

$$
\mathbf{x}=\mathbf{K}_{\mathrm{p}} \cdot \mathbf{p}
$$

where $\mathbf{K}_{\mathbf{p}}$ is the steady-state gain matrix. Given experimental data, $\mathbf{K}_{\mathbf{p}}$ matrix $\left(\in \mathfrak{R}^{60 \times 3}\right)$ can be determined from the least square regression. The regression result versus position on wafer is given in Fig. $2 a$.

Let us use the singular value decomposition (SVD) to analyze the system. The SVD decomposes the $\mathbf{K}_{\mathrm{p}}$ matrix of model into three matrices:

$$
\mathbf{K}_{\mathbf{p}}=\mathbf{U} \mathbf{\Sigma} \mathbf{V}^{T}
$$

where $\mathbf{U}$ is a orthonormal matrix $\left(\in \mathfrak{R}^{60 \times 3}\right)$ in the output side, $\mathbf{V}$ is the input orthonormal matrix $\left(\epsilon \mathfrak{R}^{3 \times 3}\right)$, and $\Sigma$ is a 
$3 \times 3$ diagonal matrix with the singular value $\left(\sigma_{i}\right)$ as the diagonal element. The ratio of the largest singular value to the smallest is the condition number $\left(\kappa=\sigma_{\max } / \sigma_{\min }\right)$ which is a quantitative indicator of closeness to singularity. After proceeding SVD, the $\mathbf{V}$ matrix is found similar to identity. Then each column of $\mathbf{U}$ matrix is plotted against position on wafer (Fig. 2b). As can be seen in the figure, the trends of the three columns in $\mathbf{U}$ matrix are quite similar to those of $K_{p}$ matrix. It indicates that the three directions are just enough to describe the characteristic of multi-zone system. The singular values sorted by number are 264,31 , and 16 , respectively, and condition number $(\kappa)$ is 16 , i.e., quite far away from a singular system. Thus, this is a well-defined multivariable system with 60 states and 3 inputs.

Because of the characteristic of a non-square property of system, it is not possible to keep all 60 outputs at their set points using only 3 inputs. Thus, non-uniformity is an inherent property of this multi-zone CMP. Given the initial surface profile $\left(y^{0}\right)$ (measured) and the desired thickness $y^{d}$, the amount needs to be removed $\left(\Delta y^{d}\right)$ can easily calculated by

$$
\Delta \mathbf{y}^{d}=\mathbf{y}^{0}-\mathbf{y}^{d}
$$

After the CMP with a polish time of $\Delta t$, the error between the desired and actual thicknesses can be expressed as

$$
\begin{aligned}
\mathbf{E} & =\mathbf{y}-\mathbf{y}^{d}=\left(\mathbf{y}^{0}-\mathbf{y}^{d}\right)-\left(\mathbf{y}^{0}-\mathbf{y}\right) \\
& =\left(\mathbf{x}^{d}-\mathbf{x}\right) \cdot \Delta \mathbf{t}
\end{aligned}
$$

Eq. (6) also indicates that the error is due to difference of actual removal rate $(\mathbf{x})$ and desired removal rate $\left(\mathbf{x}^{\mathrm{d}}\right)$. In terms of the removal rate, we have:

$$
\mathbf{x}^{d}=\mathbf{K}_{\mathrm{p}} \cdot \mathbf{p}
$$

Solving Eq. (7) by the least-square method, the input pressures are computed:

$$
\mathbf{p}=\left(\mathbf{K}_{\mathrm{p}}^{T} \mathbf{K}_{\mathrm{p}}\right)^{-1} \mathbf{K}_{\mathrm{p}}^{T} \cdot \mathbf{x}^{d}=\mathbf{K}_{\mathrm{p}}^{\dagger} \cdot \mathbf{x}^{d}
$$

where the superscript $\dagger$ denotes the pseudo-inverse. Substituting p into Eq. (3), one finally obtains:

$$
\begin{aligned}
\mathbf{E}_{\min } & =\left(\mathbf{I}-\mathbf{K}_{\mathrm{P}} \mathbf{K}_{\mathrm{p}}^{\dagger}\right) \mathbf{x}^{d} \cdot \Delta \mathbf{t} \\
= & \left(\mathbf{I}-\mathbf{K}_{\mathrm{p}} \mathbf{K}_{\mathrm{p}}^{\dagger}\right) \Delta \mathbf{y}^{d}
\end{aligned}
$$

The above equation indicates the achievable performance for this multi-zone CMP and it is characterized by the deviation of the projection matrix $\left(\mathbf{K}_{\mathbf{p}} \mathbf{K}_{\mathbf{p}}^{\dagger}\right)$ from an identity matrix. Consider the case when every entry of the vector $\Delta \boldsymbol{y}^{d}$ takes the value of 1. Fig. 3 shows the shape of the surface profile in the radial position. Since the 2 -norm of $E_{\min }$ is equal to 0.0735 , it implies an averaged error of $73.5 \AA$ for $1000 \AA$ removed.

\section{CONTROL}

In the process control notation, the measured data are the controlled variables and the manipulated variables are the three pressures applied to each zone. Therefore, this is a $60 \times 3$ multivariable control problem. The control objective is to maintain uniform surface profile using all three manipulated variables. Because this is a non-square system, it is not possible to keep all the outputs at their set points using only 3 inputs. A 2-norm-based objective function is then considered:

$$
J=\left\|\mathbf{y}^{\prime}-\mathbf{y}^{2}\right\|_{2}
$$

where $J$ is the objective function, $\mathbf{y}^{f}$ and $\mathbf{y}^{d}$ are vectors $\left(\in \mathfrak{R}^{60 \times 1}\right)$ of measured thicknesses (at end of each run, i.e., $\mathbf{y}^{f}=\mathbf{y}\left(\mathrm{t}_{\mathrm{f}}\right)$ ) and the desired thicknesses, respectively.

Some performance indices are introduced here.

i) Standard deviation $(S D)$ :

$$
S D=\sqrt{\sum_{i=1}^{\infty}\left(y_{,}^{\prime}-\bar{y}^{\prime}\right)^{2} / n}
$$

ii) $\infty$-norm of the deviation between measured thickness and the mean:

$$
\infty \text {-norm }=\left\|y^{f}-\bar{y}^{f}\right\|_{\infty}
$$

iii) The range of measured thickness:

range $=y_{\max }^{f}-y_{\min }^{f}$

iv) Non-uniformity $(N U)$ :

$$
N U=\frac{S D}{\bar{y}^{\prime}} \times 100 \%
$$

Those provide simple measures of quality after polishing. Obviously, the achievable performance defined by Eq. (9) also provides an absolute basis to evaluate control performance.

\section{I Ratio Control}

Since the process model, at least $\mathrm{Kp}$, is perfectly know, a simple control strategy can be implemented for the multizone CMP process. It is a feedforward control where the ratios of the pressures are computed from the model inverse and ratios are maintained throughout the run..

The final NU in flat case is about $5 \%$ which is acceptable in the fabrication. In other cases, the first two surface profiles with small SD value, IC1 and IC2, are regarded as finer initial conditions among the three. The indices of final status of those cases are slightly more than those of achievable status. However, their NUs are both kept within $5 \%$. The SD of final status of IC3 is reduced while the other indices are not changing much. Basically, this kind of initial surface profile is hard to handle for the system. Thus, that indicates that ECP is supported to prevent production of that profile.

\subsection{Multivariable Feedback Control}

It is based on concept of feedback control. The controller adjusts the pressure input simultaneously according to the deviation between measured thickness by sensor and set points (desired thickness). To monitor the change of surface in a polish run, therefore, an in situ sensor is required to measure copper thickness on-line and a nonsquare feedback controller necessary to generate input pressures. Fig. 4a shows the non-square system with the tall process (more output than inputs) and a fat controller (more input to the controller than the control output). The 
SVD-based approach is employed to design the inversedbased controller. First, the steady-state gain matrix is decomposed into three matrices and the multivariable system can be expressed as (Fig. 4b):

$$
\begin{aligned}
& \mathbf{Y}=\frac{1}{s} \mathbf{K}_{\mathrm{p}} \cdot \mathbf{D} \cdot \mathbf{p}=\frac{1}{s}\left(\mathbf{U} \Sigma \mathbf{V}^{\tau}\right) \mathbf{D} \cdot \mathbf{p} \\
& =\mathbf{U} \cdot \operatorname{diag}\left[\frac{\sigma_{i}}{s}\right] \cdot \mathbf{V}^{T} \cdot \mathbf{D} \cdot \mathbf{p}
\end{aligned}
$$

Next, the derivative part of the controller is taken as the inverse of $\mathbf{D}$ and the output of the PI part of the controller becomes:

$$
\mathbf{p}=\mathbf{D}^{-1} \mathbf{p}_{P t}
$$

Thus, the relationship between the $\mathrm{p}_{\mathrm{P}}$ and $\mathrm{Y}$ becomes:

$$
\mathbf{Y}=\mathbf{U} \cdot \operatorname{diag}\left[\frac{\sigma_{i}}{s}\right] \cdot \mathbf{V}^{T} \mathbf{p}_{P i}
$$

Multiply the both sides with $\mathbf{U}^{\mathbf{T}}$ matrix (recall that both $\mathbf{U}$ and $\mathrm{V}$ are orthonormal matrices, i.e., $\mathbf{U U}^{T}=I$ and $\mathbf{V}^{T} \mathbf{V}=I$ ), one obtains:

$$
\mathbf{U}^{T} \mathbf{Y}=\operatorname{diag}\left[\frac{\sigma_{i}}{s}\right] \cdot \mathbf{V}^{T} \cdot \mathbf{p}_{p l}
$$

$\mathbf{U}^{\mathrm{T}} \mathbf{Y}$ and $\mathbf{V}^{\mathrm{T}} \mathbf{p}_{\mathrm{PI}}$ correspond to the output and input in the principle directions and they are defined as $\mathbf{Y}^{*}\left(\in \Re^{3 \times 1}\right)$ and $\mathbf{p}^{*}\left(\in \mathfrak{R}^{3 \times 1}\right)$, respectively. Therefore, we are left with a decoupled square system with simple diagonal elements, $\sigma_{i} / s$. Define the diagonal matrix as $\mathbf{G}_{\mathbf{p}}{ }^{*}$, the multivariable system becomes:

$$
\mathbf{Y}^{*}=G_{p}^{*} \mathbf{p}^{*}
$$

Fig. $4 \mathrm{c}$ also shows the result of transformation. Note the diagonalizing effort is absorbed into the controller as shown in Fig. 4b. Therefore, standard multivariable control method can be applied to this decoupled square system.

Taking the flat profile as an example, Fig. 5a shows the snapshots of the surface profiles throughout the polishing process. The control performance can be seen from the responses of the manipulated variables (Fig. $5 \mathrm{~b} \mathrm{\&} \mathrm{c)} \mathrm{and}$ the tracking error (Fig. 5d). In terms of the principle output (Fig. 5d) and input (Fig. 5c), the output settles to the set point in less than 20 seconds and acceptable control performance is obtained using the SVD-based multivariable controller. For CMP process, the ultimate performance measure in the uniformity. Table 1 summarizes all the indices using the ratio control as well as the multivariable feedback control. Comparison is made with respect to the achievable performance (Table 1). Results clearly indicate that the multivariable feedback control leads to the performance close to the achievable performance while the ratio control shows $4-15 \%$ deviations from the achievable one. More importantly, the seemingly complex control system can be design systematic manner with rather standard feedback design methodology.

\section{CONCLUSION}

In this work, a systematic modeling and control system design approaches are proposed for a multi-zone CMP system. The multi-zone CMP is intended to reduce the within-wafer non-uniformity (WIWNU) by manipulating different pressures across the radial position. This leads to a non-square multivariable system, a $60 \times 3$ system for the example studied. In the modeling phase, two $2^{3}$ full factorial experimental design is carried out and steady-state gain matrix is obtained from the lease square regression followed by including the dynamic element associated with each input. For multivariable control, two control strategies with different degrees of complexity are proposed. First, a simple ratio control is designed based on the pseudoinverse of the process model and, provided with the initial surface profile, the input pressures are computed and the ratios are maintained throughout the polishing run. The results show that, while giving reasonable uniformity, the performance is a little short from the achievable performance. Next, an on-line multivariable control system is designed. The singular value decomposition (SVD) is used to project the input and output in the principle directions and, therefore, the controllers can be designed in a reduced dimension $(3 \times 3)$ for a decoupled system. Then, the diagonal controllers are transformed back to the true input/output spaces. This significantly reduces the engineering effort in control system design. Results show that achievable control performance can be maintained using the SVD-based multivariable controllers.

\section{ACKNOWLEDGEMENT}

SJS, AJS and CCY thank National Science Council of Taiwan for the financial support under the grant NSC 922214-E002-032.

\section{REFERENCES}

[1] Preston, F. W., "The theory and design of plate glass polishing machines", J. Soc. Glass Technology, 11, 214-256 (1927). 
Table 1. Different uniformity measures of wafer surfaces before and after CMP using ratio control, multivariable feedback control and achievable performance

\begin{tabular}{|c|c|c|c|c|c|}
\hline \multicolumn{2}{|c|}{ Surface profile } & Flat & $\mathrm{ICl}$ & $\mathrm{IC} 2$ & IC3 \\
\hline \multirow{4}{*}{ Initial } & SD & 0 & $62 . \overline{21}$ & 88.47 & 159.4 \\
\hline & $\infty$-norm & 0 & 378 & 636 & 603 \\
\hline & range & 0 & 444 & 714 & 734 \\
\hline & $\mathrm{NU}(\%)$ & 0 & 0.71 & 1 & 1.15 \\
\hline \multirow{5}{*}{ Achievable } & $\left\|E_{\min }\right\|_{2}$ & 727 & 535 & 698 & 1170 \\
\hline & SD & 95.46 & 70.3 & 91.61 & 153.6 \\
\hline & $\infty$-norm & 189 & 142 & 321 & 457 \\
\hline & range & 363 & 272 & 541 & 802 \\
\hline & $\mathrm{NU}(\%)$ & 4.77 & 3.51 & 4.58 & 7.68 \\
\hline \multirow{2}{*}{ Final } & $\|E\|$ & 786 & 618 & 740 & 1218 \\
\hline & $\|\boldsymbol{L}\|_{2}$ & 751 & 579 & 703 & 1201 \\
\hline \multirow{3}{*}{$\begin{array}{l}\text { (Top: ratio } \\
\text { control }\end{array}$} & & 101 & 77.5 & 97 & 157 \\
\hline & SD & 95.23 & 70.04 & 91.52 & 153.33 \\
\hline & & 225 & 223 & 330 & 444 \\
\hline \multirow{5}{*}{$\begin{array}{l}\text { Bottom: } \\
\text { multivariable } \\
\text { feedback } \\
\text { control })\end{array}$} & $\infty$-norm & 189 & 143 & 322 & 455 \\
\hline & & 379 & 345 & 528 & 778 \\
\hline & range & 362 & 273 & 541 & 800 \\
\hline & NUl $1 \%$ & 5.01 & $3 . \overline{83}$ & 4.83 & 7.72 \\
\hline & $N U(\%)$ & 4.7 & 3.45 & 4.55 & 7.53 \\
\hline \multirow{2}{*}{\multicolumn{2}{|c|}{ Relative error ${ }^{*}$}} & 8.12 & 15.51 & 6.02 & 4.1 \\
\hline & & 3.3 & 8.22 & 0.72 & 2.65 \\
\hline
\end{tabular}

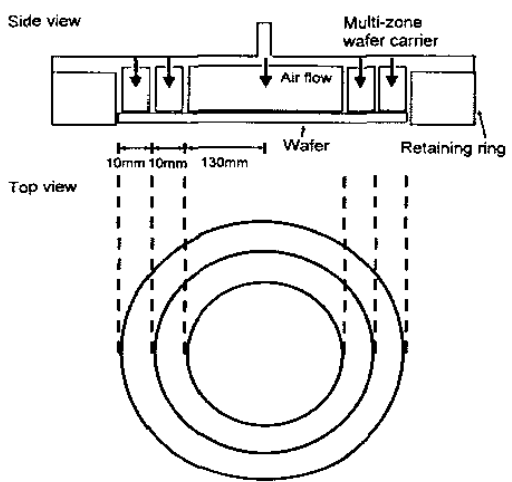

Fig. 1. Configuration of multi-zone CMP.
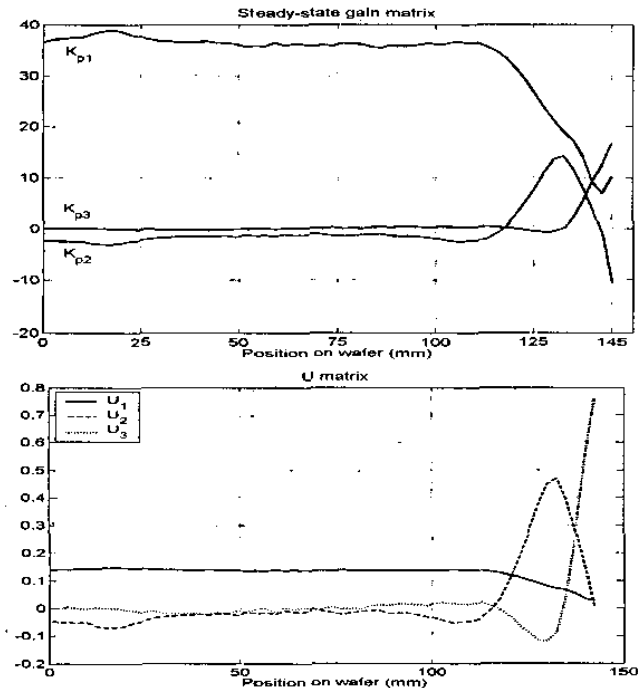

Fig. 2. Steady-state gain vectors and vectors of the $U$ matrix from SVD

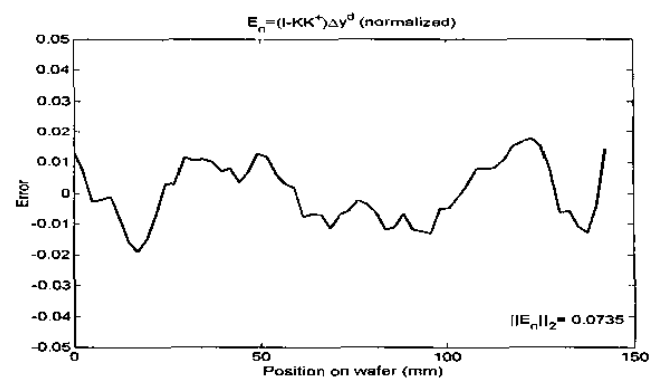

Fig. 3. The error vector of achievable performance by assuming input vector of unity.

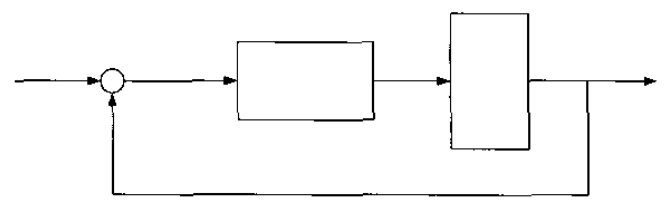

(a) Original non-square system

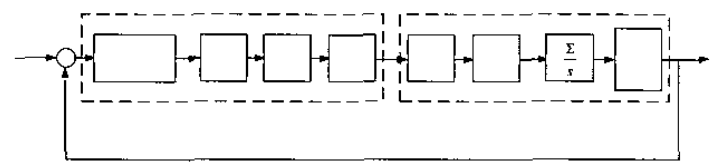

(b) SVD of the Process and Controller

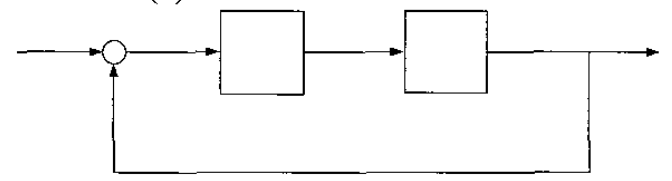

(c) Equivalent Decoupled System

Fig. 4. Evolution of the SVD-based design and corresponding block diagrams.

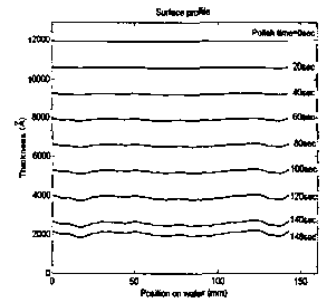

(a)

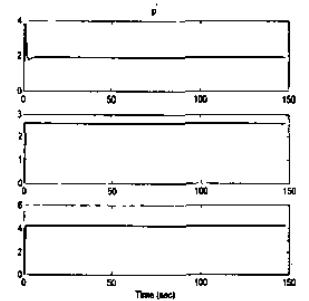

(c)

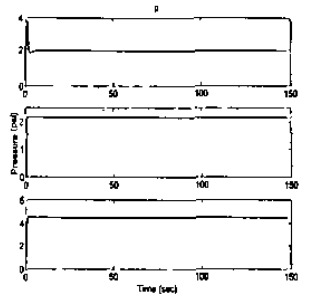

(b)

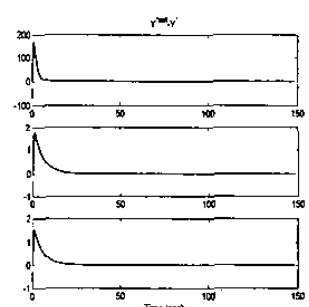

(d)
Fig. 5. Results of multivariable feedback control for incoming wafer with flat profile: (a) snap shots of surface profile, (b) pressures, (c) principal input, and (d) error in the principle direction. 